\title{
Comparative study of the interfaces of graphene and hexagonal boron nitride with
} silver

Garnica, Manuela; Schwarz, Martin; Ducke, Jacob; He, Yuanqin; Bischoff, Felix; Barth, Johannes V.; Auwarter, Willi; Stradi, Daniele

Published in:

Physical Review B

Link to article, DOI:

10.1103/PhysRevB.94.155431

Publication date:

2016

Document Version

Publisher's PDF, also known as Version of record

Link back to DTU Orbit

Citation (APA):

Garnica, M., Schwarz, M., Ducke, J., He, Y., Bischoff, F., Barth, J. V., Auwarter, W., \& Stradi, D. (2016).

Comparative study of the interfaces of graphene and hexagonal boron nitride with silver. Physical Review $B$, 94(15), [155431]. https://doi.org/10.1103/PhysRevB.94.155431

\section{General rights}

Copyright and moral rights for the publications made accessible in the public portal are retained by the authors and/or other copyright owners and it is a condition of accessing publications that users recognise and abide by the legal requirements associated with these rights.

- Users may download and print one copy of any publication from the public portal for the purpose of private study or research.

- You may not further distribute the material or use it for any profit-making activity or commercial gain

- You may freely distribute the URL identifying the publication in the public portal 


\title{
Comparative study of the interfaces of graphene and hexagonal boron nitride with silver
}

\author{
Manuela Garnica, ${ }^{*}$ Martin Schwarz, Jacob Ducke, Yuanqin He, Felix Bischoff, \\ Johannes V. Barth, and Willi Auwärter \\ Physik-Department E20, Technische Universität München, James-Franck-Strasse 1, 85748 Garching, Germany \\ Daniele Stradi \\ Department of Micro- and Nanotechnology, Center for Nanostructured Graphene, Technical University of Denmark, \\ DK-2800 Kgs. Lyngby, Denmark
}

(Received 23 May 2016; revised manuscript received 19 August 2016; published 18 October 2016)

\begin{abstract}
Silver opens up interesting perspectives in the fabrication of complex systems based on heteroepitaxial layers after the growth of a silicene layer on its (111) face has been proposed. In this work we explore different synthesis methods of hexagonal boron nitride $(h$-BN) and graphene sheets on silver. The resulting layers have been examined by high-resolution scanning tunneling microscopy. A comparison of the interfacial electronic band structure upon growth of the distinct two-dimensional (2D) layers has been performed by scanning tunneling spectroscopy and complementary first-principle calculations. We demonstrate that the adsorption of the 2D layers has an effect on the binding energy of the Shockley state and the surface potential by lowering the local work function. These effects are larger in the case of graphene where the surface state of $\operatorname{Ag}(111)$ is depopulated due to charge transfer to the graphene. Furthermore, we show that the electronic properties of the $h$-BN/silver system can be tuned by employing different thicknesses of silver ranging from a few monolayers on $\mathrm{Cu}(111)$ to the single crystal Ag substrate.
\end{abstract}

DOI: 10.1103/PhysRevB.94.155431

\section{INTRODUCTION}

In recent years, silver surfaces emerged as a promising platform for the synthesis of atomically thin materials after the growth of silicene has been proposed [1], which presents potential advantages for future device applications [2]. The synthesis and investigation of other 2D materials on $\operatorname{Ag}(111)$ at the atomic scale opens up possibilities to design $2 \mathrm{D}$ heterostructures that can provide access to fundamental physics and an improved flexibility to realize devices [3]. As an initial step for these potential applications, a reliable synthesis of single- and few-layer 2D materials becomes crucial. In particular, the chemical vapor deposition (CVD) technique has been shown advantageous to fabricate ultrathin, large-scale graphene and $h$-BN sheets on reactive metals like $\mathrm{Ru}, \mathrm{Rh}$, Ir, $\mathrm{Co}, \mathrm{Ni}$ and Pd [4-8]. However, the low catalytic activity of noble metals as $\mathrm{Ag}$ and $\mathrm{Au}$ impairs the growth of graphene [9] and $h$-BN $[10,11]$ by standard CVD procedures. As an alternative method, atomic deposition has been successfully used for the growth of graphene [12,13]. However, the synthesis of $h$-BN on silver is still challenging [10].

The pertaining interfaces are of great interest and importance. We can classify them depending on the interaction strength between the 2D layer and the metal [6]. Silver is considered a weakly interacting system where the electronic properties of both the 2D layer and the silver substrate would remain practically unperturbed. In particular, a unique feature of the close-packed surfaces of noble metals is the presence of a surface state with nearly free electron gas characteristics. Scanning tunneling microscopy (STM) is a powerful tool to probe these states, which are confined in perpendicular direction to the surfaces. Relevant and quantitative information can

*manuela.garnica@tum.de be extracted from the study of the evolution and modification of these states by the adsorption of gases [14-16], molecules [17-22], ionic films [23], and 2D honeycomb layers [24-26]. On the other hand, scanning tunneling spectroscopy (STS) has been exploited to investigate the unoccupied states of ultrathin layers on metals, in particular, the image potential states [24,27-29]. They are bound to a solid by the response of the substrate to the presence of the electron and kept outside the surface by the reflective properties of the substrate. Experimentally, one can access these states in the fieldemission regime by STS and they are thus known as field emission resonances (FERs) [30]. The evolution of the FERs can provide quantitative information on changes in the local electrostatic surface potential which manifest as local work function variation. In this work we present an investigation of the modification of the surface states on the (111) face of a silver crystal after the growth of graphene and boron nitride yielding information about the interfaces. We track back the modification of the Shockley-type surface state to charge transfer and local work function modifications upon the formation of the $2 \mathrm{D}$ layers.

\section{METHODS}

The experiments were performed in a custom-designed ultrahigh vacuum (UHV) system, equipped with a commercial low-temperature CreaTec STM. The base pressure was below $2 \times 10^{-10}$ mbar. The $\operatorname{Ag}(111)$ single crystal surface was prepared by several cycles consisting of sputtering by a $800 \mathrm{eV}$ $\mathrm{Ar}^{+}$ion beam followed by annealing at $900 \mathrm{~K}$. All STM images were taken at $5 \mathrm{~K}$ in constant-current mode and the differential conductance $(d I / d V)$ spectra were recorded using a lock-in amplifier $\left(f=969 \mathrm{~Hz}, \Delta V_{\mathrm{rms}}=18 \mathrm{mV}\right)$. We measured the FERs with the feedback loop connected at a current setpoint 
of $0.8 \mathrm{nA}$. The STM images were processed using the WSxM software [31].

The simulations of graphene and $h-\mathrm{BN}$ on $\mathrm{Ag}(111)$ were performed using density functional theory (DFT) as implemented in SIESTA [32]. The electronic exchange-correlation energy was described using the Perdew-Burke-Ernzerhof (PBE) functional [33]. The ionic cores were described by Trullier-Martins pseudopotentials [34]. The real-space grid was set to a plane-wave equivalent of $200 \mathrm{Ry}$. The one-electron Kohn-Sham orbitals were expanded in a basis of double$\zeta$ numerical atomic orbitals (NAOs) including polarization functions. The NAOs confinement radii $r_{c}$ of the $\mathrm{B}, \mathrm{C}$, and $\mathrm{N}$ $s(p)$ shells were set to 5.16 (6.30), 4.41 (5.38), and $3.97 \AA$ (4.73 $\AA$ ), respectively. For $\mathrm{Ag}$, the confinement radii for the $s(d)$ shells were set to $5.37 \AA(5.97 \AA)$, and an additional set of $s$-like diffuse functions $\left(r_{c}=9.0 \AA\right)$ was added to the topmost layer basis set to account correctly for the electronic properties of the $\operatorname{Ag}(111)$ surface [35]. The graphene/ $\operatorname{Ag}(111)$ $(h$-BN/Ag(111)) system was simulated by using a 27-layer symmetric Ag slab, with the graphene $(h-\mathrm{BN})$ placed at both sides of the slab. The mismatch between the graphene ( $h$-BN) and the $\operatorname{Ag}(111)$ surface was accounted for by matching a $2 \times 2$ graphene $(h$-BN) supercell to a $\sqrt{3} \times \sqrt{3} \operatorname{Ag}(111)$ supercell [36,37]. The resulting Brillouin zone was sampled using a Monkhorst-Pack [38] regular grid of $24 \times 24 \times 1 k$ points, and the electronic occupancies were determined using a Fermi-Dirac smearing of $25 \mathrm{meV}$.

\section{RESULTS}

\section{A. Synthesis of 2D layers on silver}

Submonolayer coverages of $h$-BN were prepared by thermal decomposition of borazine $(\mathrm{HBNH})_{3}$ (Katchem, Czech Republic) assisted by an ion gun. The ion gun used in the standard surface preparation in UHV is used to create a beam of ionized precursors which is accelerated towards the hot sample [9]. This procedure enhances the thermal decomposition of the precursor compared to a normal CVD procedure and allows us to grow patches of $h$-BN even at moderate temperatures. Figure 1(a) shows an STM image recorded after exposing the Ag substrate at $900 \mathrm{~K}$ to $2700 \mathrm{~L}$ of borazine using a beam energy of $300 \mathrm{eV}$. The structural quality of the $h$-BN islands is highlighted by the inset of Fig. 1(a). At this particular setpoint the atomic corrugation of the 2D layer is superimposed with a periodicity corresponding to a moiré pattern. The lateral periodicity of the moiré is $1 \mathrm{~nm}$ with a small apparent corrugation of less than $10 \mathrm{pm}$, which does not depend on the bias voltage. Even if this is the most common moiré superstructure for our samples, the presence of several rotational domains due to the weak interaction between $h$-BN and silver has previously been reported [10].

Nevertheless, this procedure is not efficient for obtaining large coverages of $h$-BN. Since a well controlled growth of $h$-BN has been achieved on $\mathrm{Cu}(111)[28,39]$ we used an alternative strategy to grow $h$-BN on silver. First, we prepare the desired coverage of $h-\mathrm{BN}$ on a $\mathrm{Cu}(111)$ crystal by CVD following previous recipes [28]. Thereupon we intercalate silver by $e$-beam evaporation while keeping the sample at $573 \mathrm{~K}$. This temperature has been shown to be suitable for a homogeneous intercalation of silver on graphene on $\operatorname{Ir}(111)$ [25] and for cobalt intercalation on $h$-BN on different metals [40,41]. Figure 1(b) shows an STM image of a partially $h$-BN covered $\mathrm{Ag} / \mathrm{Cu}(111)$ surface. Previous studies of silver growth on $\mathrm{Cu}(111)$ showed a Stranski-Krastanov growth for temperatures higher than $300 \mathrm{~K}[42,43]$. A $9 \times 9$ superstructure is formed due to the mismatch between $\mathrm{Ag}$ and $\mathrm{Cu}$ lattices and is clearly observable by STM and LEED (see the Supplemental Material Fig. S1 [44]). The superstructure has a periodicity of $2.4 \mathrm{~nm}$ as can be seen in the high resolution STM image of Fig. S1(a) [44].

For the graphene growth, it has been shown that the atomic carbon evaporation from a graphite rod gives rise to graphene islands [12]. Following this procedure, Figs. 1(c) and 1(d) depict large scale STM images of graphene on a $\mathrm{Ag}(111)$ surface. The graphene coverage is controlled by the carbon deposition time and flux. The graphene layers exhibit extended areas of a superstructure with a hexagonal order and a periodicity of $1.7 \mathrm{~nm}$, which corresponds to a moiré pattern where Ag and graphene are aligned [inset of Fig. 1(c)]. We also found small domains of graphene appearing flat [see Fig. S3(c) [44]].
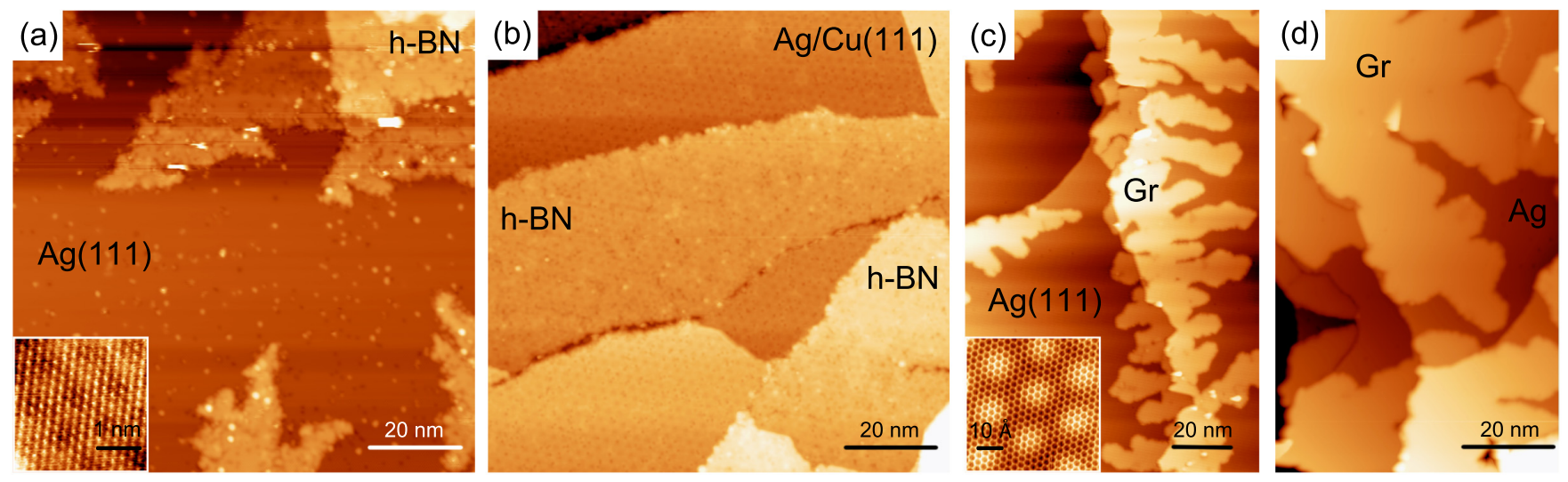

FIG. 1. (a) Overview STM image of $h-\mathrm{BN}$ on $\operatorname{Ag}(111)\left(V_{\mathrm{b}}=1 \mathrm{~V}, I_{\mathrm{t}}=2 \mathrm{nA}\right)$. The inset of (a) shows an atomically resolved STM image of the $h$-BN layer. (b) STM image of $h$-BN flakes on 3 monolayers (ML) of Ag on $\mathrm{Cu}(111)\left(V_{\mathrm{b}}=1 \mathrm{~V}, I_{\mathrm{t}}=0.1 \mathrm{nA}\right)$. Large scale STM images after $\mathrm{C}$ deposition to generate graphene (Gr) islands on the Ag crystal at $900 \mathrm{~K}$ for (c) $15 \min \left(V_{\mathrm{b}}=1 \mathrm{~V}, I_{\mathrm{t}}=0.1 \mathrm{nA}\right)$ and $(\mathrm{d}) 30 \mathrm{~min}\left(V_{\mathrm{b}}=1 \mathrm{~V}\right.$, $I_{\mathrm{t}}=0.08 \mathrm{nA}$ ). The inset of (c) shows an atomically resolved STM image of the moiré of graphene grown on $\mathrm{Ag}(111)$. 

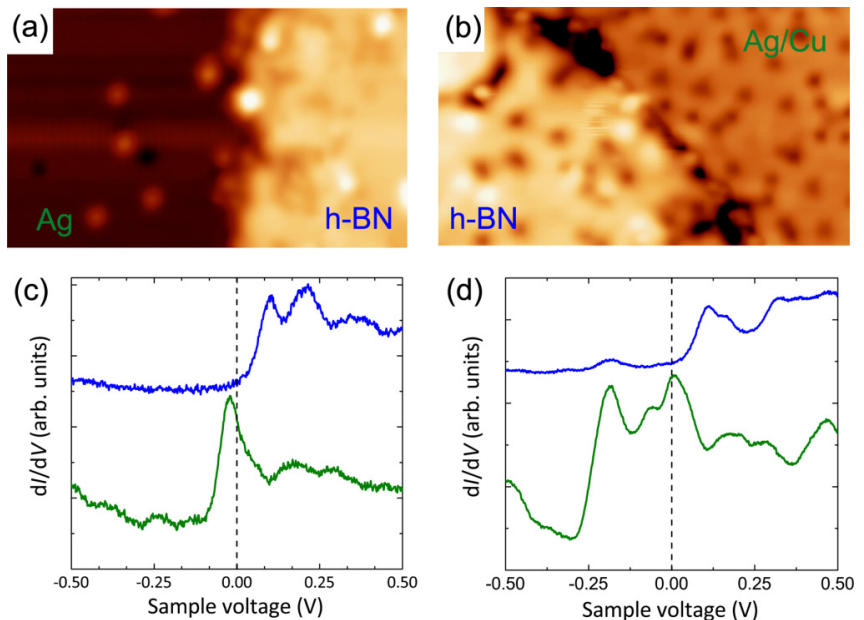

(d)
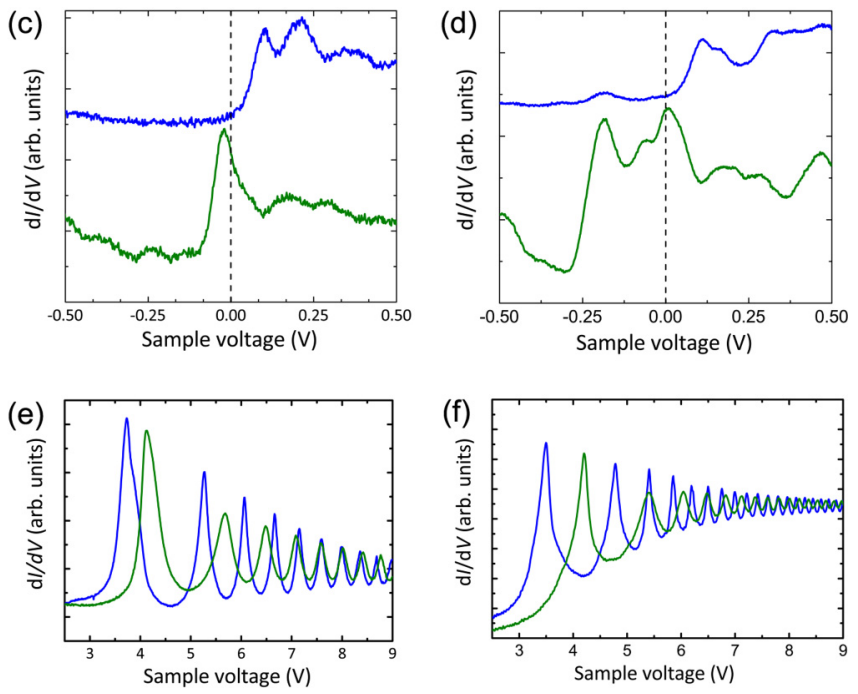

FIG. 2. STM images of (a) $h-\mathrm{BN} / \operatorname{Ag}(111)\left(V_{\mathrm{b}}=1 \mathrm{~V}, I_{\mathrm{t}}=\right.$ $0.8 \mathrm{nA})$ and (b) $h-\mathrm{BN} / \mathrm{Ag} / \mathrm{Cu}(111)\left(V_{\mathrm{b}}=0.5 \mathrm{~V}, I_{\mathrm{t}}=0.1 \mathrm{nA}\right)$. Individual $d I / d V$ curves (c) around the Fermi level and (e) in the field emission regime measured on bare $\mathrm{Ag}(111)$ (green) and $h$-BN/Ag (blue) areas. Individual $d I / d V$ curves (d) around the Fermi level and (f) in the field emission regime measured on $\mathrm{Ag} / \mathrm{Cu}(111)$ (green) and $h-\mathrm{BN} / \mathrm{Ag} / \mathrm{Cu}(111)$ (blue) areas.

\section{B. Spectroscopic characterization}

To get further insight into the interaction between the 2D layers and the metallic substrate, we performed scanning tunneling spectroscopy (STS) investigations. Figures 2(a) and 2(b) show STM images of the interface between $h$-BN and $\mathrm{Ag}(111)$ and $\mathrm{Ag} / \mathrm{Cu}(111)$, respectively. In the case of $h-\mathrm{BN}$ on $\mathrm{Ag}(111)$ [Fig. 2(c)], the spectrum measured around the Fermi level on $\operatorname{Ag}(111)$ (green line) shows the classic steplike feature in agreement with earlier findings [45,46]. This step corresponds to an increased local density of states (LDOS) due to the presence of the two-dimensional surface state of silver. For the $h$-BN areas (blue line) the onset of the surface state is up-shifted by $119 \mathrm{mV}$ compared to the bare $\mathrm{Ag}(111)$. It also presents features ascribed to the scattering at the boundaries of the $h$-BN island. The same effect is observed in the case of $h-\mathrm{BN}$ on $\mathrm{Ag} / \mathrm{Cu}(111)$ [Fig. 2(d)]. Here the band minimum appears at $-235 \mathrm{mV}$ on the $\mathrm{Ag} / \mathrm{Cu}(111)$ areas and at $79 \mathrm{mV}$ on the $h$-BN areas. This results in a total shift of $314 \mathrm{mV}$. In both cases, instead of a smooth shift of the surface resonance going from the bare $\mathrm{Ag}(111)$ to the 2D layer, the transition is abrupt and the surface state vanishes in the vicinity of the step edge (see Fig. S3 [44]). Even if the energy position of the spectral feature in the $h$-BN islands is similar in both systems, there is a large difference in the onset on the $\mathrm{Ag} / \mathrm{Cu}(111)$ areas with respect to the bare silver. Previous work has shown that the $\mathrm{Ag}$ film thickness on $\mathrm{Cu}(111)$ determines the energy shift of the
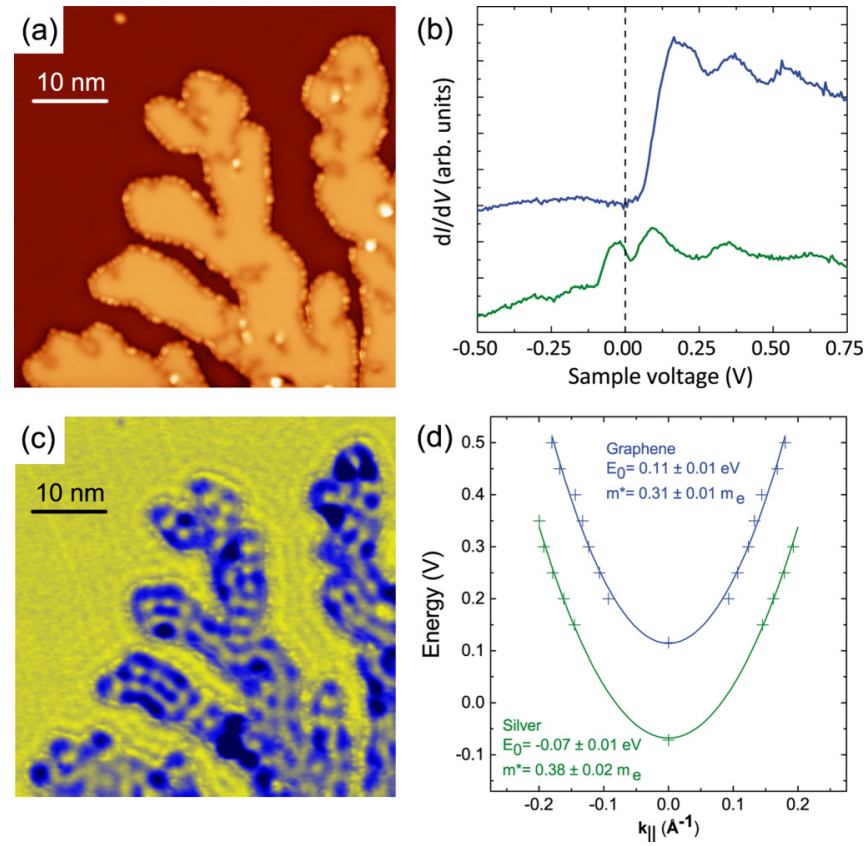

FIG. 3. (a) STM image of a graphene island on $\operatorname{Ag}(111)\left(V_{\mathrm{b}}=\right.$ $0.3 \mathrm{~V}, I_{\mathrm{t}}=0.3 \mathrm{nA}$ ). (b) $d I / d V$ spectra measured on graphene (blue) and bare silver (green). (c) $d I / d V$ map at $0.3 \mathrm{~V}$ acquired simultaneously with image (a). (d) Dispersion data $E\left(k_{\|}\right)$of the surface state band (crosses) and corresponding fits (solid lines) for the bare silver and graphene covered areas. The experimental data point at $k_{\|} \neq 0$ are extracted from a series of $d I / d V$ maps at different energies.

Shockley-type surface state [47]. Accordingly, we conclude that the copper surface is covered by $3 \mathrm{ML}$ of silver (see Fig. S1 [44]).

A change in the local work function in the presence of $h$-BN is also observed. Figures 2(e) and 2(f) show the evolution of the FERs on the $h-\mathrm{BN} / \mathrm{Ag}(111)$ and $h-\mathrm{BN} / \mathrm{Ag} / \mathrm{Cu}(111)$ systems where a clear shift is discernible. A very similar behavior has already been observed in $h$-BN on other metals $[28,29]$, however in these cases the moiré provides a corrugated potential. The local work function was deduced from the binding energy of the FERs (see Fig. S4 [44]). The $\mathrm{Ag}(111)$ work function is decreased by $\sim 0.35 \mathrm{eV}$ and the $\mathrm{Ag} / \mathrm{Cu}(111)$ work function by $\sim 0.65 \mathrm{eV}$ upon the $h$-BN layer formation.

Figures 3(a) and 3(b) show the topography and spectroscopic signature of a graphene island on $\operatorname{Ag}(111)$. As in the case of $h$-BN on silver, we observe an up-shift $(\sim 180 \mathrm{mV})$ of the surface state of $\operatorname{Ag}(111)$ in the graphene layer. In addition, we measured $d I / d V$ maps at different voltages. These maps show the standing waves patterns with different wavelengths on bare $\mathrm{Ag}(111)$ and at graphene areas. The analysis of the corresponding fast Fourier transform (FFT) images of the maps provide qualitative information about the effective mass $m^{*}$ of the electrons. The fitting of the parabolic dispersion of the surface state to the 2D free electron gas model $E=E_{0}+$ $(\hbar k)^{2} / 2 m^{*}$ results in the following values for the bare silver and graphene on $\mathrm{Ag}(111): E_{0}=-0.07 \pm 0.01 \mathrm{eV}, m^{*}=0.38 \pm$ $0.02 m_{e}$ and $E_{0}=0.11 \pm 0.01 \mathrm{eV}, m^{*}=0.31 \pm 0.01 m_{e}$, respectively. The effective mass is mainly determined by the metal considering that the weak bonding of the layers does not 

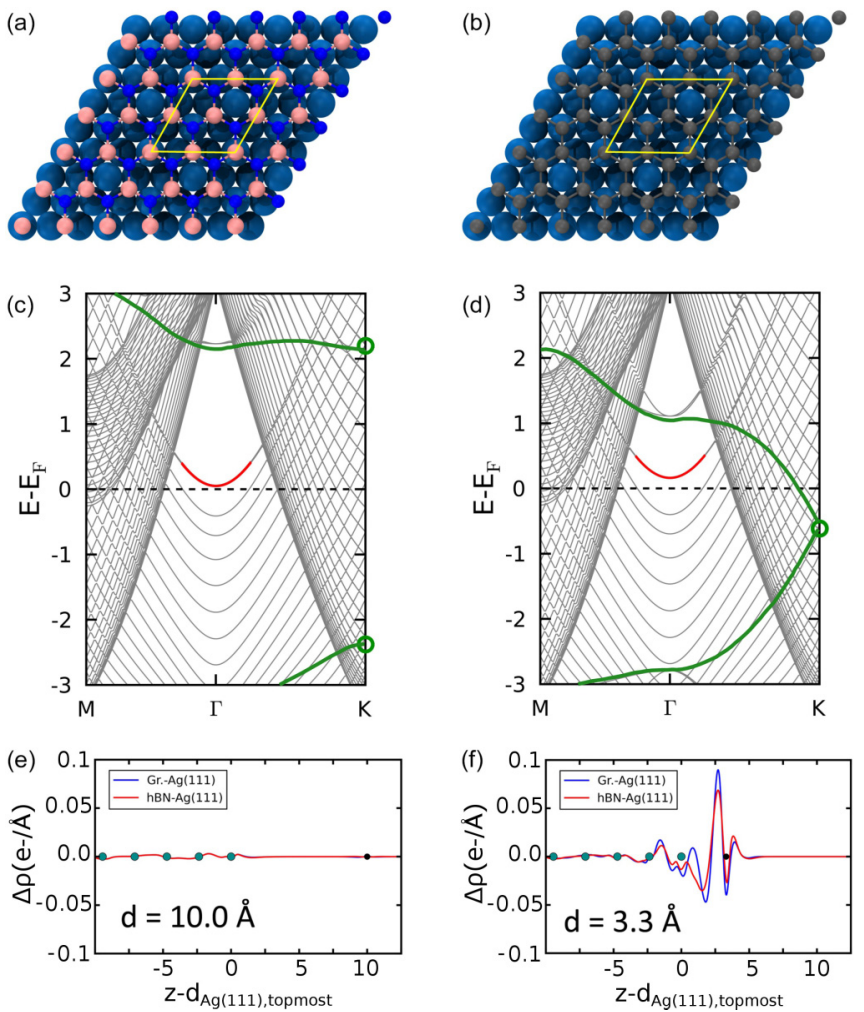

FIG. 4. Top view of the (a) $h$-BN/Ag(111) and (b) graphene/ $\operatorname{Ag}(111)$ structure used in the calculations (the supercell is highlighted in yellow). (c) $h-\mathrm{BN} / \mathrm{Ag}(111)$ and (d) graphene/Ag(111) electronic band structure. In the band structure plots, the Shockley surface state is highlighted in red. The green circles mark the position of $h$-BN bands (green lines) and the Dirac point at $K$. Distance dependence of the charge redistribution $\Delta \rho$ upon adsorption of graphene (blue lines) and $h$-BN (red lines) on $\operatorname{Ag}(111)$ for (e) $d=10.0 \AA$, and (f) $d=3.3 \AA$. $\Delta \rho$ has been integrated in the $X Y$ plane. The black and dark cyan dots indicate the positions of the graphene $(h-\mathrm{BN})$ and $\mathrm{Ag}(111)$ layers, respectively.

strongly affect the metal surface. However, for an insulator a slight increase in the effective mass of the surface band was observed [23]. On the other hand, in the case of graphene on $\mathrm{Au}(111)$ [26] it remains unchanged or even decreases for a graphene layer grown on a copper crystal [24], which effect was attributed to an increased surface corrugation.

\section{Theoretical characterization}

To get a comprehensive understanding of the experimental data and to fully characterize the electronic structure, we performed DFT calculations. Figures 4(a) and 4(b) show the structure of $h$-BN/Ag(111) and graphene/ $\operatorname{Ag}(111)$ used in the calculations where the supercell is highlighted in yellow for both systems. In both cases, the calculated band structure reproduces the silver surface state shift observed in the experiments. In particular, we obtain a shift of $227.8 \mathrm{mV}(115 \mathrm{mV})$ for graphene/Ag(111) $(h$-BN/Ag(111)) at a distance of $d=3.3 \AA$ from the surface, which should be close to the exact binding distance for graphene/Ag [48]. At this distance, the calculated adsorption energies per two atoms (CC or $\mathrm{BN}$ ) for graphene/ $\operatorname{Ag}(111)$ and $h-\mathrm{BN} / \mathrm{Ag}(111)$ are $E_{\mathrm{ads}}^{\mathrm{Gr} / \mathrm{Ag}(111)}=-132.5 \mathrm{meV} / \mathrm{CC}$ and $E_{\text {ads }}^{h-\mathrm{BN} / \mathrm{Ag}(111)}=-112.5 \mathrm{meV} / \mathrm{BN}$, respectively. We notice how the value of $E_{\mathrm{ads}}^{\mathrm{Gr} / \mathrm{Ag}(111)}$ is strikingly close to that reported in the literature using either the random phase approximation (RPA) [48] or nonlocal exchange-correlation functionals [37] $\left(E_{\mathrm{ads}}^{\mathrm{Gr} / \mathrm{Ag}(111)}=-156 \mathrm{meV} / \mathrm{CC}\right)$, although this is the result of an error compensation, as dispersion interactions are not included explicitly in the present calculations.

The surface state shift strongly depends on the equilibrium distance (see Fig. S5 [44]). The present large interlayer distance is a consequence of the weak interaction between the $h-\mathrm{BN}$ (graphene) and the $\mathrm{Ag}(111)$ and seems to allow for the confinement of the silver surface state at the interface. This finding is in contrast to strongly interacting systems, e.g., nanomesh structures such as $h$-BN/Rh(111) $\left(d_{\mathrm{N}-\mathrm{Rh}}=2.1 \AA\right)$ [49], graphene islands grown on $\mathrm{Ni}(111)\left(d_{\mathrm{Ni}-\mathrm{Gr}}=2.1 \AA\right)$ [50], and graphene grown on $\operatorname{Ru}(0001)\left(d_{\mathrm{Ru}-\mathrm{Gr}}=2.4 \AA\right)$ [27]. In particular, in the latter two cases, the surface state of the metal evolves into an interface state as a result of a considerable interaction between $\mathrm{C}$ and metal atoms.

Figures 4(e) and 4(f) show the distance dependence of the charge redistribution $\Delta \rho$ upon adsorption of graphene and $h$-BN on $\operatorname{Ag}(111)$. Note that at the equilibrium distance (3.3 $\AA$ ) the charge redistribution mainly affects the interface and is slightly higher in the case of graphene $/ \operatorname{Ag}(111)$ than in $h$-BN/Ag(111).

\section{DISCUSSION}

First, we compare the results for $h$-BN on $\mathrm{Ag}(111)$ and on $3 \mathrm{ML} \mathrm{Ag} / \mathrm{Cu}(111)$. Table I shows the experimental surface state shifts and work function changes for these systems. Both values are larger in the case of $h-\mathrm{BN} / \mathrm{Ag} / \mathrm{Cu}(111)$. Whereas the $h$-BN layers appear similar in the STM images [see Figs. 2(a) and 2(b)], they are distinct from the electronic point of view. Before the silver intercalation, the surface state band onset of the bare $\mathrm{Cu}(111)$ is at -440 and at $-334 \mathrm{mV}$ for the $h$ $\mathrm{BN} / \mathrm{Cu}(111)$ regions $\left(\Delta E_{\mathrm{SS}}=106 \mathrm{mV}\right)$. The work function is reduced and, contrary to $h$-BN/Ag(111), spatially modulated following the moiré superstructure $(-0.84 \mathrm{eV}$ in the valleys and $-1.14 \mathrm{eV}$ on the hills) [28]. Once the silver intercalates, the surface state band minimum is shifted smoothly to higher values in the $\mathrm{Ag} / \mathrm{Cu}(111)$ areas (e.g., at $-235 \mathrm{mV}$ for $3 \mathrm{ML}$ of $\mathrm{Ag}$ ) [47]. On the $h$-BN regions, the surface state depopulates and the band onset appears at similar energies as for $h$-BN

TABLE I. Comparison of the observed surface state shifts and work function changes. $\Delta E_{\mathrm{SS}}^{\text {expt }}$ and $\Delta E_{\mathrm{SS}}^{\text {theo }}$ are the experimental and calculated surface state shift of the silver. $\Delta \Phi$ is the 2 D layer induced change of work function with respect to the pristine surface. $E_{\text {ads }}^{\text {theo }}$ corresponds to the adsorption energy per two atoms (CC or BN).

\begin{tabular}{lccc}
\hline \hline & $h$-BN/Ag & $h$-BN/3 ML Ag/Cu & $\mathrm{Gr} / \mathrm{Ag}$ \\
\hline$\Delta E_{\mathrm{SS}}^{\text {expt }}(\mathrm{mV})$ & 119 & 314 & 180 \\
$\Delta E_{\mathrm{SS}}^{\text {theo }}(\mathrm{mV})$ & 115 & - & 227.8 \\
$\Delta \Phi(\mathrm{eV})$ & -0.35 & -0.65 & $-0.68[36]$ \\
$E_{\text {ads }}^{\text {theo }}(\mathrm{meV})$ & -112.5 & - & -132.5 \\
\hline \hline
\end{tabular}


on $\operatorname{Ag}(111)$ [see Figs. 2(c) and 2(d)]. Thus, the shift of the surface state induced by the $h$-BN is considerably larger on $\mathrm{Ag} / \mathrm{Cu}(111)(314 \mathrm{mV})$ than in the case of $\mathrm{Ag}(111)(119 \mathrm{mV})$. It is interesting to note that the $\mathrm{Ag}$ thickness allows us to tune the properties of the system in a controlled manner.

Inspection of Table I also shows a larger shift of the Shockley surface state band for graphene/Ag(111) than for $h$-BN/Ag(111). We attribute this behavior to the charge redistribution at the interface [Fig. 4(f)]. It shows the same tendency as the surface state shift, which is larger in the case of graphene on $\mathrm{Ag}(111)$. Following the charge redistribution and the empirical rule postulated by Ziroff et al. [17] for physisorbed overlayers on noble metal (111) surfaces, the up-shift of the surface state energy can be correlated to the adsorption energy per surface area. Consequently, we might expect a higher interaction between graphene and silver than between boron nitride and silver. Indeed, the calculated adsorption energies reproduce this trend correctly, as at a similar adsorption distance, graphene/ $\operatorname{Ag}(111)$ is predicted to be more stable than h-BN/Ag(111) by $20 \mathrm{meV} / \mathrm{CC}$. In the case of $h-\mathrm{BN}$ on $3 \mathrm{ML} \mathrm{Ag} / \mathrm{Cu}(111)$ the interaction is expected to be even larger.

Comparing the calculated band structure of Figs. 4(c) and 4(d), we can extract further information related to the modification of the electronic structure of the 2D layers. Due to the presence of a wide gap in the $h$-BN band structure, the electronic states of silver around the Fermi level are not perturbed fundamentally upon adsorption. In particular, $h$-BN/Ag(111) presents a $4.52 \mathrm{eV}$ gap at the $K$ point [see green circles in Fig. 4(c)]. Neither the calculations nor the experimental data provide evidence for BN-related states in the gap, as expected for a weakly interacting system [51]. In the same way, in the case of the graphene, the characteristic Dirac cone at the $K$ point is preserved but slightly shifted below the Fermi level indicating a physisorbed $n$-doped graphene [see the green circle in Fig. 4(d)]. The calculated shift is $0.57 \mathrm{eV}$. This observation is in agreement with the blueshift of the $G$ band in the Raman spectrum observed experimentally [12] and is due to the charge transfer between graphene and the metal consistent with the depopulation of the surface state.
Considering only the direction of the charge transfer, we might expect an increase in the work function. However, as can be seen in Table I, our results, as well as the previous calculations [36,52], show a work function decrease upon the graphene and $h$-BN adsorption. This effect is explained by an interfacial dipole induced by Pauli repulsion upon adsorption of the physisorbed layers, dominating over the contribution of the charge transfer [52].

\section{CONCLUSIONS}

In conclusion, we have investigated the electronic structure of a silver surface upon the growth of graphene and $h$-BN. In both cases, the surface state of the metal is still detected and its onset is shifted towards higher energies. The analysis of the binding energy shift of the Shockley state is used to shed light on the relative strength of the interaction between the epitaxial layer and the metallic substrate. We find that the shift can be controlled by the silver thickness in the $h-\mathrm{BN} / \mathrm{Ag} / \mathrm{Cu}(111)$ system and that the charge transfer contributes to the depopulation of the band in the case of the graphene. Contrary to the direction of the charge transfer a decrease of the work function of both systems is observed. The charge redistribution and the modification of the surface dipole upon adsorption of the 2D layer is behind this effect. This crucial information will be important for the further design and investigation of a heteroepitaxial growth of 2D lateral heterostructures on silver.

\section{ACKNOWLEDGMENTS}

We thank F. Kraus, S. Rudel, and M. König (TUM, Department Chemie) for help with the borazine transfer. This work is supported by the European Research Council Consolidator Grant NanoSurfs (No. 615233) and the Munich Center for Advanced Photonics (MAP). M.G. would like to acknowledge the H2020-MSCA-IF-2014 programme. We are grateful for support from the H. C. Ørsted-COFUND postdoc program at DTU (D.S.). W.A. acknowledges funding by the Deutsche Forschungsgemeinschaft via a Heisenberg professorship.
[1] P. Vogt, P. De Padova, C. Quaresima, J. Avila, E. Frantzeskakis, M. C. Asensio, A. Resta, B. Ealet, and G. Le Lay, Phys. Rev. Lett. 108, 155501 (2012).

[2] L. Tao, E. Cinquanta, D. Chiappe, C. Grazianetti, M. Fanciulli, M. Dubey, A. Molle, and D. Akinwande, Nat. Nanotechnol. 10, 227 (2015).

[3] A. K. Geim and I. V. Grigorieva, Nature (London) 499, 419 (2013).

[4] W. Auwärter, T. Kreutz, T. Greber, and J. Osterwalder, Surf. Sci. 429, 229 (1999).

[5] Y. Gamo, A. Nagashima, M. Wakabayashi, M. Terai, and C. Oshima, Surf. Sci. 374, 61 (1997).

[6] A. B. Preobrajenski, M. L. Ng, A. S. Vinogradov, and N. Mårtensson, Phys. Rev. B 78, 073401 (2008).

[7] A. Varykhalov and O. Rader, Phys. Rev. B 80, 035437 (2009).
[8] Y. Murata, E. Starodub, B. B. Kappes, C. V. Ciobanu, N. C. Bartelt, K. F. McCarty, and S. Kodambaka, Appl. Phys. Lett. 97, 143114 (2010).

[9] A. J. Martínez-Galera, I. Brihuega, and J. M. Gómez-Rodríguez, Nano Lett. 11, 3576 (2011).

[10] F. Müller, S. Hüfner, H. Sachdev, R. Laskowski, P. Blaha, and K. Schwarz, Phys. Rev. B 82, 113406 (2010).

[11] L. Camilli, E. Sutter, and P. Sutter, 2D Mater. 1, 025003 (2014).

[12] B. Kiraly, E. V. Iski, A. J. Mannix, B. L. Fisher, M. C. Hersam, and N. P. Guisinger, Nat. Commun 4, 2804 (2013).

[13] I. Hernández-Rodríguez, J. M. García, J. A. Martín-Gago, P. L. D. Andrés, and J. Méndez, Diam. Relat. Mater. 57, 58 (2015).

[14] J.-Y. Park, U. D. Ham, S.-J. Kahng, Y. Kuk, K. Miyake, K. Hata, and H. Shigekawa, Phys. Rev. B 62, R16341(R) (2000). 
[15] F. Forster, G. Nicolay, F. Reinert, D. Ehm, S. Schmidt, and S. Hüfner, Surf. Sci. 532-535, 160 (2003).

[16] H. Hövel, B. Grimm, and B. Reihl, Surf. Sci. 477, 43 (2001).

[17] J. Ziroff, P. Gold, A. Bendounan, F. Forster, and F. Reinert, Surf. Sci. 603, 354 (2009).

[18] Y. Pennec, W. Auwärter, A. Weber-Bargioni, A. Schiffrin, A. Riemann, and J. V. Barth, Nat. Nanotechnol. 2, 99 (2007).

[19] R. Temirov, S. Soubatch, A. Luican, and F. S. Tautz, Nature (London) 444, 350 (2006).

[20] K. Kanazawa, M. Nakamura, H. Huang, A. Taninaka, and H. Shigekawa, Surf. Sci. 632, L1 (2015).

[21] G. Rojas, S. Simpson, X. Chen, D. A. Kunkel, J. Nitz, J. Xiao, P. A. Dowben, and A. Enders, Phys. Chem. Chem. Phys. 14, 4971 (2012).

[22] F. Klappenberger, D. Kühne, W. Krenner, I. Silanes, A. Arnau, F. J. García de Abajo, D. Abajo, S. Klyatskaya, M. Ruben, and J. V Barth, Nano Lett. 9, 3509 (2009).

[23] J. Repp, G. Meyer, and K.-H. Rieder, Phys. Rev. Lett. 92, 036803 (2004).

[24] S. M. Hollen, G. A. Gambrel, S. J. Tjung, N. M. Santagata, E. Johnston-Halperin, and J. A. Gupta, Phys. Rev. B 91, 195425 (2015).

[25] W. Jolie, F. Craes, and C. Busse, Phys. Rev. B 91, 115419 (2015).

[26] P. Leicht, L. Zielke, S. Bouvron, R. Moroni, E. Voloshina, and L. Hammerschmidt, ACS Nano 8, 3735 (2014).

[27] B. Borca, S. Barja, M. Garnica, D. Sánchez-Portal, V. M. Silkin, E. V. Chulkov, C. F. Hermanns, J. J. Hinarejos, A. L. Vázquez de Parga, A. Arnau, P. M. Echenique, and R. Miranda, Phys. Rev. Lett. 105, 036804 (2010).

[28] S. Joshi, D. Ecija, R. Koitz, M. Iannuzzi, A. P. Seitsonen, J. Hutter, H. Sachdev, S. Vijayaraghavan, F. Bischoff, K. Seufert, J. V. Barth, and W. Auwärter, Nano Lett. 12, 5821 (2012).

[29] F. Schulz, R. Drost, S. K. Hämäläinen, T. Demonchaux, A. P. Seitsonen, and P. Liljeroth, Phys. Rev. B 89, 235429 (2014).

[30] G. Binnig, K. H. Frank, H. Fuchs, N. Garcia, B. Reihl, H. Rohrer, F. Salvan, and A. R. Williams, Phys. Rev. Lett. 55, 991 (1985).

[31] I. Horcas, R. Fernández, J. M. Gómez-Rodríguez, J. Colchero, J. Gómez-Herrero, and A. M. Baro, Rev. Sci. Instrum. 78, 013705 (2007).

[32] J. M. Soler, E. Artacho, J. D. Gale, A. Garcia, J. Junquera, P. Ordejon, and D. Sanchez-Portal, J. Phys.: Condens. Matter 14, 2745 (2002).

[33] J. P. J. Perdew, K. Burke, and M. Ernzerhof, Phys. Rev. Lett. 77, 3865 (1996).
[34] N. Troullier and J. L. Martins, Phys. Rev. B 43, 1993 (1991).

[35] S. García-Gil, A. García, N. Lorente, and P. Ordejón, Phys. Rev. B 79, 075441 (2009).

[36] G. Giovannetti, P. A. Khomyakov, G. Brocks, V. M. Karpan, J. van den Brink, and P. J. Kelly, Phys. Rev. Lett. 101, 026803 (2008).

[37] I. Lončarić and V. Despoja, Phys. Rev. B 90, 075414 (2014).

[38] H. J. Monkhorst and J. D. Pack, Phys. Rev. B 13, 5188 (1976).

[39] A. B. Preobrajenski, A. S. Vinagradov, and N. Mårtensson, Surf. Sci. 582, 21 (2005).

[40] W. Auwärter, M. Muntwiler, T. Greber, and J. Osterwalder, Surf. Sci. 511, 379 (2002).

[41] A. B. Preobrajenski, M. L. Ng, N. A. Vinogradov, A. S. Vinogradov, E. Lundgren, A. Mikkelsen, and N. Mårtensson, Nano Lett. 9, 2780 (2009).

[42] W. McMahon, E. S. Hirschorn, and T.-C. Chiang, Surf. Sci. Lett. 279, L231 (1992).

[43] A. Bendounan, Y. F. Revurat, B. Kierren, F. Bertran, and V. Y. Yurov, Surf. Sci. 496, L43 (2002).

[44] See Supplemental Material at http://link.aps.org/supplemental/ 10.1103/PhysRevB.94.155431 for detailed explanation of the silver reconstruction on $\mathrm{Cu}(111)$, the growth of graphene by CVD, the evolution of the surface state across a step, the field emission resonance and the energy shift as a function of the distance between graphene ( $h$-BN) and $\mathrm{Ag}(111)$.

[45] J. Li, W.-D. Schneider, R. Berndt, O. R. Bryant, and S. Crampin, Phys. Rev. Lett. 81, 4464 (1998).

[46] J. Kliewer, R. Berndt, E. V. Chulkov, V. M. Silkin, P. M. Echenique, and S. Crampin, Science 288, 1399 (2000).

[47] M. Wessendorf, C. Wiemann, M. Bauer, M. Aeschlimann, M. A. Schneider, H. Brune, and K. Kern, Appl. Phys. A 78, 183 (2004).

[48] T. Olsen and K. S. Thygesen, Phys. Rev. B 87, 075111 (2013).

[49] R. Laskowski, P. Blaha, and K. Schwarz, Phys. Rev. B 78, 045409 (2008).

[50] A. Garcia-Lekue, T. Balashov, M. Olle, G. Ceballos, A. Arnau, P. Gambardella, D. Sanchez-Portal, and A. Mugarza, Phys. Rev. Lett. 112, 066802 (2014).

[51] A. B. Preobrajenski, S. A. Krasnikov, A. S. Vinogradov, M. L. Ng, T. Käämbre, A. A. Cafolla, and N. Mårtensson, Phys. Rev. B 77, 085421 (2008).

[52] P. A. Khomyakov, G. Giovannetti, P. C. Rusu, G. Brocks, J. van den Brink, and P. J. Kelly, Phys. Rev. B 79, 195425 (2009). 